\title{
Cyclic dominance and biodiversity in well-mixed populations
}

\author{
Jens Christian Claussen ${ }^{1}$ and Arne Traulsen ${ }^{2}$ \\ ${ }^{1}$ Institut für Theoretische Physik und Astrophysik, Christian-Albrechts Universität, Olshausenstraße 40, 24098 Kiel, Germany \\ ${ }^{2}$ Max-Planck-Institute für Evolutionsbiologie, August-Thienemann-Str. 2, 24306 Plön, Germany
}

(Dated: June 19, 2007; revised October 26, 2007)

\begin{abstract}
Coevolutionary dynamics is investigated in chemical catalysis, biological evolution, social and economic systems. The dynamics of these systems can be analyzed within the unifying framework of evolutionary game theory. In this Letter, we show that even in well-mixed finite populations, where the dynamics is inherently stochastic, biodiversity is possible with three cyclic dominant strategies. We show how the interplay of evolutionary dynamics, discreteness of the population, and the nature of the interactions influences the coexistence of strategies. We calculate a critical population size above which coexistence is likely.
\end{abstract}

PACS numbers: 87.23.-n, 89.65.-s 02.50.Ey

Coevolution with cyclic dominance can lead to oscillatory, chaotic and stochastic dynamics. For example, such cycles can be found in biology [1, 2, 3, 4, 5, 6, 6, 7] or in social and economic systems [8, 9]. The simplest example for such a cyclic dominance is the well known children's game rock-paperscissors, where rock crushes scissors, scissors cuts paper and paper wraps rock. Different biological realizations of this system have been observed. For example, a cyclic dominance of three male strategies has been reported in lizards [1, 2]: Orange-throated males establish large territories holding several females. These populations are invaded by males with yellow-striped throats, which do not contribute to the defense of the territory but sneak on the females. Such a population of yellow-striped males can be invaded by blue-throated males, which defend territories large enough to hold one female which they defend against sneakers. Once yellow-striped sneakers are rare, it is advantageous to defend a large territory with several females and the cycle starts with orange-throated males again. Another example is the competition between different strains of E.coli. Kerr et al. [3, 4] observed that cyclic dominance leads to biodiversity in spatial systems, whereas two strategies go extinct in mixed systems after a short time. Such systems have been analyzed by evolutionary game theory in great mathematical detail $[10,11,12,13,14,15,16]$. Based on the replicator dynamics describing the dynamics in an infinite population, general conclusions on the nature of the payoff matrix can be made from the observation of fast extinction. How does this picture change if we tackle the more realistic case of stochastic dynamics in a finite population [17, 18, 19]? Depending on population size and the underlying microscopic process [20, 21, 22, 23], the resulting dynamics can be very different from the the replicator equation results.

Definition of the model. - We first concentrate on the simplest example of cyclic rock-paper-scissors dynamics, in which all three strategies are equivalent [24]. Thus, the game is defined by three payoffs: (i) the payoff against a dominated strategy (set to 1). (ii) the payoff against a dominant strategy $-s$, which we assume to be negative. (iii) the payoff for a tie (set to 0 ). Thus, we obtain the payoff matrix

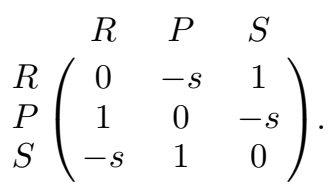

Only for the standard choice $s=1$, we have a zero-sum game. An intuitive understanding how $s$ influences the game can be obtained from discussing two relevant cases. Large values of $s$ will make it successful to avoid losing, best done with staying with the majority. In this case, a mixed equilibrium is unstable and ultimately, only one strategy will survive. For $s \approx 0$, it is more important to win occasionally, such that the mixed equilibrium can become stable.

Let us first recall the evolutionary dynamics in an infinite population described by the replicator dynamics [10]. In the replicator equation, the frequency (abundance) $x_{k}$ of strategy $k$ changes proportional to its payoff $\pi_{k}$,

$$
\dot{x}_{k}=x_{k}\left(\pi_{k}-\langle\pi\rangle\right) .
$$

Here $\langle\pi\rangle$ is the average payoff in the population. We denote the frequencies of $\mathrm{R}, \mathrm{P}, \mathrm{S}$ as $x, y, z$, respectively, with $x+$ $y+z=1$. With the standard assumption that payoffs are determined from interactions with a representative subset of the population, we find $\pi_{R}=z-s y, \pi_{P}=x-s z$, and $\pi_{S}=$ $y-s x$. The average payoff is given by $\langle\pi\rangle=(1-s)(x y+$ $x z+y z)$ vanishing for the zero-sum game with $s=1$. Eq. (2) has an interior fixed point $\boldsymbol{p}=\left(\frac{1}{3}, \frac{1}{3}, \frac{1}{3}\right)$. The determinant of the payoff matrix $d=1-s^{3}$ determines the dynamics of the system [10]. For $d>0$, which is the case for $s<1$,

$$
H=-x y z=-x y(1-x-y)
$$

is a Lyapunov function with $\dot{H}<0$ and the interior fixed point $\boldsymbol{p}$ is asymptotically stable. For $d<0(s>1), \boldsymbol{p}$ is unstable and the attractor of the system approaches a heteroclinic cycle at the boundary of the simplex $S_{3}$. Finally, for the zero-sum game with $s=1$ and $d=0$ the function (3) is a constant of motion, and the system infinitely oscillates around $\boldsymbol{p}$. This is not a purely theoretical exercise: Experiments indicate $d>0$ 
for the Lizard system [2] and $d<0$ for the E.coli system [3]. In theoretical approaches, often the restriction to $s=1$ is made, although this is a special non-generic case [15, 16]. It has been argued that in this case, limited mobility in a spatially extended system can promote biodiversity. However, for $s<1$ biodiversity is likely even in a well-mixed population if the population is larger than $N_{c}$. In this Letter, we give an analytical estimate for this critical population size, depending on $s$ and the update mechanism. For $s=1$ the average time to extinction scales linearly with the population size $N$ [23]. For $s>1$, one expects faster extinction, as the stochastic drift and the instability of the interior fixed point act jointly. In the most interesting case of $s<1$, both forces work in opposite directions: For $N \rightarrow \infty$, the fixed point $\boldsymbol{p}$ is stable, but a finite $N$ leads to a drift towards the simplex boundaries.

Evolutionary processes. - To study dynamics in finite populations, we study microscopic stochastic processes for the dynamics, which lead to macroscopic equations of motion for large populations [8, 20, 21]. As the microscopic dynamics may depend on the system, the respective biological or behavioral setup may require different interaction and competition processes. To demonstrate the robustness of our results, we consider different birth-death processes: the frequencydependent Moran process (MO) [17, 18, 19], and local twoparticle interaction processes [20, 21, 27, 28, 29].

In finite populations with $i R$-players, $j P$-players, and $N-i-j S$-players, the payoffs can be calculated from the equations for infinite $N$ by replacing $x \rightarrow i /(N-1)$, $y \rightarrow j /(N-1)$, and $z \rightarrow(N-i-j) /(N-1)$. By dividing by $N-1$ and setting the payoff for ties zero, we formally exclude self-interactions. For the average payoff, we have $\langle\pi\rangle=\frac{i}{N} \pi^{R}+\frac{j}{N} \pi^{P}+\frac{N-i-j}{N} \pi^{S}$.

In the frequency-dependent Moran process, an individual reproduces proportional to its fitness. Then, the offspring replaces a randomly selected individual [30]. The transition probabilities of the possible six hopping events are given by $\left(T^{R S}:=T^{R \rightarrow S}\right)$

$$
\begin{aligned}
T^{R S} & =\frac{1}{2} \frac{1-w+w \pi^{S}}{1-w+w\langle\pi\rangle} \frac{i}{N} \frac{N-i-j}{N} \\
T^{S R} & =\frac{1}{2} \frac{1-w+w \pi^{R}}{1-w+w\langle\pi\rangle} \frac{i}{N} \frac{N-i-j}{N}
\end{aligned}
$$

and $T^{S P}, T^{P S}, T^{P R}, T^{R P}$ are obtained by cyclic permuta- tion of $(R, P, S)$ and $(i, j, N-i-j)$. Fitness is a convex combination of a constant background fitness (set to 1) and the payoff. The parameter $w>0$ controls the intensity of selection; random genetic drift is obtained for $w \rightarrow 0$. For better comparison with the processes below, we have introduced an additional factor $1 / 2$.

Selecting an individual proportional to fitness requires knowledge about every payoff in the population. In many cases, it is more realistic to assume that competition occurs locally between two individuals. One process of this type is the local update (LU) process [20, 21], where one individual $b$ is selected randomly for reproduction, compares with annother randomly chosen individual $a$, and changes strategy with probability $\frac{1}{2}\left(1+w\left(\pi_{a}-\pi_{b}\right)\right)$ [31]. In general, the reproductive fitness can depend in a nonlinear way on the payoff difference between two competing agents. as in the Fermi process (FP) [27, 28, 29].

We now unify the processes by means of a reproductive function,

$$
\begin{aligned}
\Phi_{\mathrm{MO}}(b \rightarrow a) & =\frac{1}{2} \frac{1-w+w \pi_{a}}{1-w+w\langle\pi\rangle} \\
\Phi_{\mathrm{LM}}(b \rightarrow a) & =\left(1+w\left(\pi_{a}-\langle\pi\rangle\right)\right) / 2 \\
\Phi_{\mathrm{LU}}(b \rightarrow a) & =\left(1+w\left(\pi_{a}-\pi_{b}\right)\right) / 2 \\
\Phi_{\mathrm{FP}}(b \rightarrow a) & \left.=\left[1+\exp \left(-w\left(\pi_{a}-\pi_{b}\right)\right)\right)\right]^{-1} .
\end{aligned}
$$

By (7) we introduce a linearized Moran (LM) process as first order approximation of the MO in the limit of weak selection, $w \rightarrow 0$. For $w=0$, selection is neutral and the four processes are identical with random drift. Note that for the Moran processes, $\Phi(b \rightarrow a)$ depends on the average payoff, whereas for the other processes two individual payoffs are involved. The transition probabilities become

$$
T^{b a}=\Phi(b \rightarrow a) N_{a} N_{b} / N^{2} .
$$

For all processes, $\Phi(b \rightarrow a)$ considers a two-particle (birthdeath) process where an individual with fitness $\pi_{a}$ compares with the average fitness $\langle\pi\rangle$ or with annother individual $\pi_{b}$.

Average drift. - For the replicator equation of the symmetric RPS dynamics, Eq. (3) defines a constant of motion. As we are interested in the finite-size corrections, we can use $H$ as an observable for the distance to the interior fixed point. For the processes defined above, the transition probabilities allow to calculate the average change of the constant of motion within the simplex $(1 \leq i, j \leq N-1)$ as [32]

$$
\begin{aligned}
\langle\Delta H\rangle=\frac{2}{N^{5}} & \sum_{i=1}^{N-1} \sum_{j=1}^{N-i-1}\left[i j(N-i-j)\left(T^{R S}+T^{S R}+T^{S P}+T^{P S}+T^{P R}+T^{R P}\right)\right. \\
& \quad-(i-1) j(N-i-j+1) T^{R S}-(i+1) j(N-i-j-1) T^{S R}-i(j+1)(N-i-j-1) T^{S P} \\
& \left.\quad-i(j-1)(N-i-j+1) T^{P S}-(i+1)(j-1)(N-i-j) T^{P R}-(i-1)(j+1)(N-i-j) T^{R P}\right] .
\end{aligned}
$$

These expressions give the exact average drift. For the two linear processes, we can approximate the average drift by replacing 
the sums by integrals. Using $x=i / N, y=j / N$, and $z=1-x-y$, we find in the continuum limit

$$
\begin{aligned}
\langle\Delta H\rangle= & -\frac{2}{N} \int_{0}^{1} \mathrm{~d} x \int_{0}^{1-x} \mathrm{~d} y\left[y(x-z)\left(T^{R S}-T^{S R}\right)+x(z-y)\left(T^{S P}-T^{P S}\right)+z(y-x)\left(T^{P R}-T^{R P}\right)\right] \\
& +\frac{2}{N^{2}} \int_{0}^{1} \mathrm{~d} x \int_{0}^{1-x} \mathrm{~d} y\left[y\left(T^{R S}+T^{S R}\right)+x\left(T^{S P}+T^{P S}\right)+z\left(T^{P R}+T^{R P}\right)\right] .
\end{aligned}
$$

From this expression, we can perform a comparison of $\langle\Delta H\rangle$ for the different processes. The neutral case as well as the linear cases are analyzed below analytically, in Fig. 1 all processes are compared numerically.

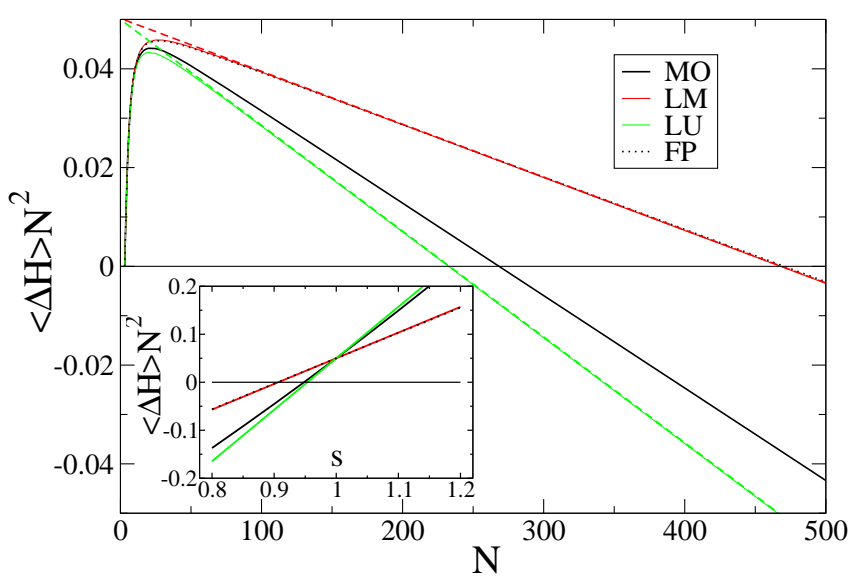

FIG. 1: (Color online.) Drift reversal for the four processes; the Linearized Moran and Fermi process coincide within line thickness. The main panel shows the scaling with population size $N$ for fixed $w=0.45$ and $s=0.8$. Full lines and the dotted line show the numerical solutions of Eq. 111. The dashed lines are the analytical expansions Eqs. (15) and (17). For small $N$, the continuum approximation to the integral leads to deviations. Inset: Average drift for varying payoff matrix with fixed $w=0.45$ and $N=1000$. The processes intersect in $\Delta H=1 /\left(20 N^{2}\right)$ at $s=1(d=0)$. For $N \rightarrow \infty$, this reduces to the expectation based on the replicator equation, $\Delta H=0$ in $d=0$. The intersection with the horizontal line indicates the drift reversal in finite populations.

For neutral evolution, $w=0$, all terms of type $T^{R S}-T^{S R}$ vanish, and we have $T^{R S}=T^{S R}=x z / 2, T^{S P}=T^{P S}=$ $y z / 2, T^{P R}=T^{R P}=x y / 2$, i.e. for $w=0$,

$$
\langle\Delta H\rangle=\frac{2}{N^{2}} \int_{0}^{1} \mathrm{~d} x \int_{0}^{1-x} \mathrm{~d} y \frac{6 x y z}{2}=\frac{1}{20 N^{2}} .
$$

Now we consider the linear terms in $w$. For the linear local update, we find $T^{R S}-T^{S R}=w x z\left(\pi^{S}-\pi^{R}\right)$, thus

$$
T^{R S}-T^{S R}=w x z((y-z)+s(y-x)) .
$$

Due to $\Phi_{L U}(b \rightarrow a)+\Phi_{L U}(a \rightarrow b)=1$, the terms of order $N^{-2}$ in Eq. (12) cancel. In first and second order of $w$, we have

$$
\langle\Delta H\rangle_{\mathrm{LU}}=\frac{1}{20 N^{2}}-\frac{1-s}{420 N} w .
$$

For the linearized Moran process, the contributions of the average payoff vanish for the drift term, and the calculation above reproduces with an additional factor $1 / 2$, i.e. $T^{R S}-$ $T^{S R}=\frac{w}{2} x z\left(\pi^{S}-\pi^{R}\right)$. For the diffusion term, we find

$$
T^{R S}+T^{S R}=x z\left(1+\frac{w}{2}\left(\pi^{S}+\pi^{R}-2\langle\pi\rangle\right)\right)
$$

and cyclic permutations. Thus, we get an additional contribution to the diffusion. In summary, we have

$$
\langle\Delta H\rangle_{\mathrm{LM}}=\frac{1}{20 N^{2}}-\frac{1-s}{420 N}\left(\frac{1}{2}-\frac{1}{N}\right) w .
$$

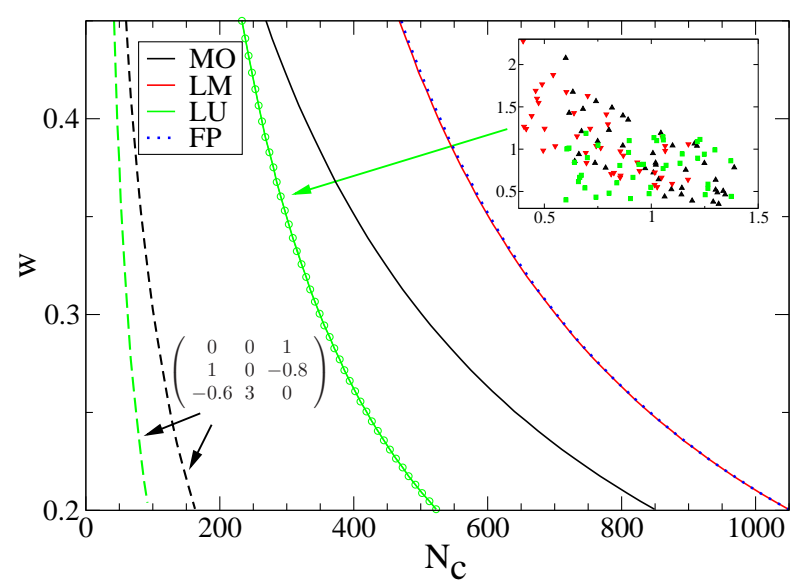

FIG. 2: (Color online.) Critical population size: Intensity of selection $w$ versus critical population size $N_{c}$, where $\Delta H$ changes its sign for fixed $s=0.8$. For $N>N_{c}$ the drift is towards the internal fixed point. The dashed lines refer to the Lizard's payoff matrix [1] for MO and LU. Under strong selection $w=1$ the LU reverses at $N_{c}=20$. For the restricted random matrix ensemble (green circles, see text) under LU $N_{c}$ coincides with the case of eq.1 Each symbol corresponds to a single random payoff matrix. (inset: scatter plot of payoff entries in $\Delta 1^{\text {st }}, \nabla 2^{\text {nd }}, \nabla 3^{\text {rd }}$ column).

Biodiversity threshold. - Both Eqs. (15) and (17) can change their sign for $s<1$ depending on $N$. For all four processes, Fig. [1] shows the average drift for different payoffs and population sizes. All processes intersect in $+1 /\left(20 N^{2}\right)$ for $s=1$. In this case, we have a zero-sum game and the average drift is equal to neutral selection. The finite-size correction to the vanishing drift term of neutral selection arises from the difference between our microscopic processes and symplectic integrators [25, 26]. In the $N \rightarrow \infty$ limit we recover that the 
stability of the interior fixed point is governed by the sign of the determinant of the payoff matrix, $d=1-s^{3}$. For $s<1$ and $N \rightarrow \infty$, trajectories spiral inwards. In finite populations, for $s<1$ the stochastic motion can be reversed as shown in Fig. 1. The critical population size of the biodiversity threshold is $N_{c}=\frac{21}{w(1-s)}$ for the LU and $N_{c}=2+\frac{42}{w(1-s)}$ for the LM. The biodiversity threshold $N_{c}$ for the other process is computed numerically in Fig. 2. For $N>N_{c}$, the $N \rightarrow \infty$ behaviour is recovered, but for $N<N_{c}$ the interior fixed point becomes unstable and the average drift goes towards the boundaries. So far, we have worked with the specific payoff matrix Eq. (1). Next, we show that this is a more general phenomenon. The drift reversal is preserved for general cyclic payoffs with $N_{c}$ depending on the payoff matrix [33]. As an example, we consider the payoff matrix of the Lizard system [1]. In this case, we find $N_{c} \geq 20$, see Fig. 2. More general, we can also address random payoff matrices with cyclic dominance. Confining a random matrix ensemble (Fig. 2) onto a suitable 2-dim submanifold [34], we show that $N_{c}(w)$ depends only on the location of the fixed point $\boldsymbol{p}$, its normalization constant $\Sigma$ and the determinant $d$, i.e. on 4 parameters. Hence the phenomenon is generic for arbitrary cyclic payoff matrices with $d>0$.
To conclude, cyclic coevolution in biological or social dynamics highlights the importance to study finite population effects and the underlying microscopic dynamics. Recently the influence of the finiteness of the population has been widely discussed for $2 \times 2$ games [18, 19, 20, 21, 22]. But also in cyclic dynamics the finiteness of the population can modify the stability conditions derived from the replicator equation. In this Letter, we have shown that biodiversity threshold of the population size occurs in generic cyclic $3 \times 3$ games for a positive determinant of the payoff matrix. Such a positive determinant has been found in the Lizard system [1,2]. Thus, stability of the coexistence fixed point can be obtained for sufficiently large populations, preserving biodiversity even in non-spatial systems or under strong mobility. In contrast, experiments in well-mixed populations of the E.coli system indicate a negative determinant of the payoff matrix.

Here we have demonstrated how nonzero-sum payoffs can change the stability even in well-mixed systems. Thus in biological - and corresponding social or economic - systems, payoff matrix, population structure, population size and the microscopic update mechanism determine the fate of extinction or coexistence.
[1] B. Sinervo, C.M. Lively, Nature 380, 240 (1996).

[2] K.R. Zamudio and B. Sinervo, PNAS 97, 14427 (2000).

[3] B. Kerr, M.A. Riley, M.W. Feldman, and B.J.M. Bohannan, Nature (London) 418, 171 (2002).

[4] B.C. Kirkup and M.A. Riley, Nature 428, 412 (2004).

[5] A. E. Magurran and M. A. Nowak, Proc. Roy. Soc. B 246, 31 (1991).

[6] Richard Dawkins, The Selfish Gene, Oxford UP (1976).

[7] U. Bastolla, M. Lässig, S. C. Manrubia and A. Valleriani, J. Theor. Biol. 235, 521-530 (2005).

[8] D. Helbing, Physica A 181, 29 (1992); 193, 241 (1993).

[9] C. Hauert, S. De Monte, J. Hofbauer and K. Sigmund, Science 296, 1129 (2002).

[10] J. Hofbauer, K. Sigmund, Evolutionary Games and Population Dynamics, Cambridge University Press (1998).

[11] M. A. Nowak, Evolutionary Dynamics, Harvard University Press (2006).

[12] G. Szabó and G. Fáth, Phys. Rep. 446, 97 (2007).

[13] G. Szabó and T. Czárán, Phys. Rev. E 64, 042902 (2001).

[14] T. Czárán, R. F. Hoekstra, and L. Pagie, PNAS 99, 786 (2002).

[15] T. Reichenbach, M. Mobilia, and E. Frey, Nature 448, 1046 (2007).

[16] T. Reichenbach, M. Mobilia, and E. Frey, Phys. Rev. Lett. 99, 238105 (2007).

[17] P. A. P. Moran, The Statistical Processes of Evolutionary Theory, Clarendon, Oxford (1962).

[18] M. A. Nowak, A. Sasaki, C. Taylor, and D. Fudenberg, Nature 428, 646 (2004).

[19] C. Taylor, D. Fudenberg, A. Sasaki, and M. A. Nowak, Bull. Math. Biol. 66, 1621 (2004).
[20] A. Traulsen, J.C. Claussen, and C. Hauert, Phys. Rev. Lett. 95, 238701 (2005).

[21] A. Traulsen, J.C. Claussen, and C. Hauert, Phys. Rev. E 74, 011901 (2006).

[22] J.C. Claussen and A. Traulsen, Phys. Rev. E 71, 025101(R) (2005).

[23] T. Reichenbach, M. Mobilia, and E. Frey, Phys. Rev. E 74, 051907 (2006).

[24] William H. Sandholm, Population Games and Evolutionary Dynamics, MIT Press, in press.

[25] J. Hofbauer, J. Math. Biol. 34, 675 (1996).

[26] Y. Sato and J. P. Crutchfield, Phys. Rev. E 67, 015206(R) (2003).

[27] L.E. Blume, Games Econ. Behav. 5, 387 (1993).

[28] G. Szabó and C. Hauert, Phys. Rev. Lett. 89, 118101 (2002).

[29] A. Traulsen, M. A. Nowak, and J. M. Pacheco, Phys. Rev. E 74, 011909 (2006).

[30] Reproduction proportional to fitness implies that $1-w+w \pi$ must be positive. In our case, this leads to $w<1 / 2$.

[31] In Ref. [20], $w$ is replaced by $w / \Delta \pi_{\max }$.

[32] Since we are only interested in large $\mathrm{N}$, a prefactor $(N-1)(N-$ 2) $/ N^{2}$ is omitted.

[33] Using $H:=-P^{3}$ with $P=x^{p_{x}} y^{p_{y}} z^{p_{z}}$ (where $p_{x}, p_{y}$ and $p_{z}$ are the coordinates of the fixed point) and normalization constant $\Sigma$, see [10] for details.

[34] With two payoff matrix entries equiprobable within a finite interval; remaining nondiagonal entries were computed such that the coordinates of the fixed point and $\Sigma$ keep fixed values. 